\section{Revue de Sémantique et Pragmatique}

37 | 2015

Sémantique et pragmatique

\title{
Éléments de cartographie des emplois de voilà en vue d'une analyse instructionnelle
}

\author{
Gilles Col, Charlotte Danino et Julien Rault
}

\section{Q OpenEdition}

1 Journals

Édition électronique

URL : http://journals.openedition.org/rsp/1015

DOI : $10.4000 /$ rsp. 1015

ISSN : 2610-4377

Éditeur

Presses universitaires d'Orléans

Édition imprimée

Date de publication : 1 juin 2015

Pagination : 37-59

ISSN : 1285-4093

\section{Référence électronique}

Gilles Col, Charlotte Danino et Julien Rault, «Éléments de cartographie des emplois de voilà en vue d'une analyse instructionnelle », Revue de Sémantique et Pragmatique [En ligne], 37 | 2015, mis en ligne le 01 juin 2016, consulté le 23 juin 2020. URL : http://journals.openedition.org/rsp/1015; DOI : https:// doi.org/10.4000/rsp.1015

Ce document a été généré automatiquement le 23 juin 2020.

Revue de Sémantique et Pragmatique 


\title{
Éléments de cartographie des emplois de voilà en vue d'une analyse instructionnelle
}

\author{
Gilles Col, Charlotte Danino et Julien Rault
}

\section{Introduction}

L'évolution et l'expansion de certaines unités lexico-grammaticales ne laissent pas d'étonner les chercheurs travaillant dans le domaine de la sémantique. Parmi ces unités, voilà se distingue singulièrement des autres et apparaît comme un " mot de l'époque ", « un nouveau tic de langage " qui " parsème nos phrases», "s'insinue de plus en plus souvent dans nos conversations » (Pourquery 2014 : 39). L'extension de ses emplois (cette " invasion de voilà ", Pourquery, ibid) nous amène à nous demander pourquoi et comment une telle unité apparaît avec une telle fréquence dans le discours. En écoutant la vacation radio d'un skipper en plein course transatlantique ${ }^{1}$, nous avons effectivement relevé aux moins trois emplois de voilà (sur 5 occurrences) répondant à trois articulations-clé des 70 secondes de vacation : au début de son intervention, pour commencer la vacation, puis au milieu pour passer à un autre sujet et pratiquement au bout du monologue pour conclure et terminer la vacation. Cette observation nous a amenés à nous demander quels sont les critères de facilitation de son développement, et finalement pourquoi voilà est ce qu'on pourrait appeler une unité... dans le vent. Audelà de l'anecdote, nous sommes confrontés à une difficulté non résolue à ce jour : la mesure précise de la fréquence de voilà. Il ne semble pas encore possible d'effectuer une telle mesure actuellement $\mathrm{du}$ fait de son aspect avant tout oral et surtout très contemporain ; nous avons donc opté pour un travail exploratoire en nous concentrant sur la variété des emplois de voilà comme source de son expansion². 


\section{1. État de l'art sur voilà}

2 Une synthèse des différentes approches linguistiques de voilà va permettre, dans un premier temps, de montrer la grande diversité des analyses, notamment dans les entreprises de catégorisation, et donc un certain embarras du discours face à ce terme polyvalent issu d'un verbe à l'impératif (voir) et d'un déictique ${ }^{3}$. Nous reprenons ici, en guise de préambule, les éléments saillants des différents travaux consacrés à voilà, principalement sur trois niveaux : statut catégoriel, signifié et dimension discursive et textuelle. La question du statut catégoriel est la plus délicate (cf. Annexe 1 : Tableau synoptique des approches de voilà). Voilà est tantôt un adverbe (alors qu'il accepte une complémentation), une préposition (avec notamment un complément de temps - « elle est partie voilà huit ans ": ce rôle reste toutefois relativement restreint), une interjection (" voilà voilà ! J'arrive ! »), un présentatif... voire « un factif strumental épidictique » pour Damourette et Pichon (1927). D'autres propositions catégorisantes hybrides auraient pu être ajoutées à cet inventaire : ainsi, voilà mi-préposition, miadverbe chez Le Bidois (1965) ou Grevisse (1980), voilà " adverbe présentatif » pour Brunot et Bruneau (1969) ou encore voilà préposition à valeur de verbe dans Le Robert. La notion de " gallicismes » - convoquée par Léard (1992) - dit également assez bien la dimension aporétique de l'entreprise de catégorisation, les " gallicisimes " étant considérés comme les " déchets inévitables des grammaires catégorielles» (Ibid : 18). Face à une telle disparité des discours, on distinguera ceux qui opèrent une subdivision en attribuant plusieurs statuts et ceux qui se sont risqués à l'étiquetage unique, au prix de quelques contorsions et concessions. Ainsi Moignet (1969), dans une approche catégorielle sémantique, définit voilà comme "une sorte de verbe " (sans variation morphologique verbale, impersonnel, unimodal et unitemporel) mais ajoute par ailleurs une valeur discursive non-prédicative qui rapproche le terme d'" une sorte de préposition ». La récurrence de la locution " une sorte de » traduit nettement la difficulté à insérer voilà dans le cadre étroit des catégories traditionnelles. Morin (1985), sur un plan syntaxique, intègre également voilà dans la catégorie du verbe. L'emploi en interjection et en préposition n'est pas analysé mais l'auteur laisse entendre (après quelques réticences) qu'un tel usage pourrait être rattaché in fine à la catégorie globale du verbe. La terminologie la plus usuelle est celle de "présentatif " qui suppose une fonction sémantique et syntaxique a priori commune, fondée sur l'origine verbale. Une telle terminologie est évidemment peu satisfaisante dans la mesure où, syntaxiquement et sémantiquement, voilà embrasse et outrepasse une nouvelle fois chacune des extrémités de cette catégorie en étant d'un côté le plus présentatif («voilà Jean-Pierre! » : valeur monstrative liée au déictique plus saillante qu'avec « il y a » ou " c'est ») et de l'autre le moins présentatif puisqu'il peut tout à fait ne présenter aucun groupe complément (le « voilà voilà » un peu embarrassé) : voilà est alors morphologiquement dépourvu de fonctionnement verbal et peut être employé de façon autonome. La tendance à l'isolement est particulièrement fréquente aujourd'hui à l'oral (" donc euh... voilà »). Est alors invoquée la dénomination " Mot du discours ", « marqueur discursif " (Hansen 1997) ou encore " marqueur de structuration de conversation » (Auchlin, 1981). À partir de cette dichotomie, entre prédicatif et nonprédicatif, il est possible de proposer un affinement par subdivision. Grenoble et Riley (1996) décrivent une "oscillation » entre présentatifs et déictiques, Narjoux (2003) distingue le présentatif prédicatif et le présentatif déchu de sa fonction prédicative (valeur prépositionnelle et interjective) et Delahaie (2009) le « voilà présentatif » et le « 
voilà conversationnel ». Porhiel (2012) extrait le rôle prépositionnel et établit trois catégories, préposition, présentatif et marqueur de discours, lesquelles rejoignent globalement celles établies par Léard (1996). Les analyses qui s'interrogent sur le statut catégoriel et qui cherchent à unifier les distributions sous un dénominateur commun tendent plutôt vers la catégorie verbale ou bien se retranchent du côté du présentatif : les insuffisances de ces catégorisations, liées aux spécificités de voilà, apparaissent systématiquement. Le point le plus remarquable, sur un plan diachronique, réside en outre dans l'évolution globale d'un " voilà verbal » vers un " voilà présentatif » (plutôt prédicatif) avec adjonction progressive et nécessaire d'un « voilà discursif ». Sur le plan du signifié, l'étymologie peut constituer un premier point d'appui, que l'on reprenne la composante déictique ou la composante verbale. Pour Léard (1996), le verbe " voir » impliquerait une perception en cours tandis que « voilà » donnerait davantage une idée de départ : " L'ouverture à la connaissance attachée à voilà s'oppose donc à la perception en cours signifiée par voir » (Léard 1996 : 124). La valeur de base de voilà, déictique et aspectuelle, suppose alors l'idée de " pointage " qui se retrouve dans le signifié de puissance proposé (« un pointage à partir du lieu ou du moment de la parole» Léard 1996 : 145). La récurrence du terme " pointage » est significative :Bergen et Plauché (2001) srappuient également sur liétymologie impérative pour formuler une valeur de " pointing out " (la valeur initiale serait spatiale avant d)être, métaphoriquement, temporelle). De Cesare (2011) évoque (dans la communication écrite et non-littéraire) la présence dıun acte linguistique assertif particulier et reprend à nouveau le terme significatif de "pointage » auquel elle adjoint un " signal discursif ». La valeur de pointage met donc laccent sur la dimension déictique et aspectuelle (Dervillez-Bastuji, 1982 : " déixis spatiale »). Il est toutefois possible de proposer un autre trait sémantique. Ainsi, Delahaie (2009) s`appuyant sur les travaux d)Anscombre (1990 et 2001), dans une approche pragmatique et sémantique considère voilà comme un élément rétroactif (contrairement à voici) inscrivant le terme qu'il introduit dans un stéréotype. La dimension aspectuelle et déictique n'est pas écartée mais se trouve éclipsée au profit d'une dimension pragmatique qui met au premier plan l'insertion de voilà dans un ensemble d'énoncés stéréotypiques (qui pose la question du caractère prévisible ou non du référent (envisagé par Wagner en 1966). Dans le discours, voilà apparaît comme un élément de structuration, doté d'un caractère introductif/conclusif auquel s'adjoint l'idée dune appréciation, d'une synthèse, d'une évaluation. Pour Auchlin (1981) l'emploi de voilà seul, « marqueur de structuration de conversation ", permet la délimitation des énoncés mais aussi la liaison (valeur de clôture/conclusion) et devient moins un connecteur argumentatif qu'un marqueur de cohésion textuelle, donnant des indications sur le niveau de textualisation des énoncés. Oppermann-Marsaux (2006) a pu montrer, sur un plan diachronique, les trois étapes de l'évolution du terme jusqu'à la fin du XVIe siècle, depuis le présentatif situationnel (situation d'énonciation), en passant par le présentatif narratif (événements narrés) jusqu'au présentatif textuel (cotexte). Pour ce troisième cas de figure, Léard ajoute une distinction supplémentaire, lorsque voilà porte sur le cotexte mais réfère à un discours autre : il peut être alors « marqueur à valeur illocutoire » (le locuteur juge et qualifie, souvent pour montrer son désaccord, l'activité linguistique de son interlocuteur) ou " marqueur à valeur géographique » (indices de la structuration du texte). Par-delà le fonctionnement déictique, voilà joue ainsi un rôle anaphorique de cohésion textuelle. On note également l'hypothèse intéressante d'une validation ( "voilà! ») ou plutôt d'un (pseudo-)ajustement (du locuteur avec son propos, avec les propos d'un interlocuteur, 
avec la situation d'énonciation) dans la lignée des analyses de Druetta (1993) qui voyait dans "voilà voilà on vient » une marque pragmatique de validation. Cet examen rapide des discours sur voilà a pu montrer la grande variété des approches, liée à la polyvalence d'un terme qui échappe à la circonscription et fuit la catégorisation. Fonder l'analyse sur la position et la distribution offre un certain nombre de points d'appui ${ }^{4}$ mais, au-delà de ces réalisations discursives diverses, au-delà de l'analyse distributionnelle, il apparaît dans un premier temps fécond d'envisager la possibilité, sur un plan sémantique, d'une référenciation globale commune : ce qui ressort des diverses analyses est sans conteste la dimension déictique, monstrative (le " pointage ») adjointe à l'idée, exploitée par Delahaie, d'un " voilà » rétroactif fondé sur une relation stéréotypique (récupération d'informations). C'est pourquoi nous nous proposons maintenant de sonder, à partir d'un corpus restreint, la possibilité d'une instruction sémantique, forme d'infra-valeur dynamique prenant en considération les caractéristiques les plus récurrentes et les plus saillantes que sont le pointage, la validation, le stéréotype.

\section{Le corpus}

3 Afin de cerner le comportement de voilà, nous avons opté pour un premier corpus qui nous sert de fenêtre d'observation et de grille de lecture pour analyser des données plus importantes 5 . Ce corpus est un corpus écrit composé de 231 exemples répartis entre de la transcription de discours oral issu de la thèse de Mélanie Petit (2009), du roman Le journal d'une femme de chambre d'Octave Mirbeau (1900) et de textes et titres de presse écrite. L'annotation du corpus est manuelle et collective, et elle a été réalisée par les auteurs de l'article et les membres de la thématique Discours et Cognition (DisCo) du laboratoire FoReLL ${ }^{6}$. Nous avons utilisé le logiciel d'annotation Analec développé au laboratoire LaTTiCe par Bernard Victorri ${ }^{7}$. Pour analyser voilà avec Analec et en tenant compte des différents emplois et valeurs de l'unité, nous avons défini le schéma d'annotation suivant : 


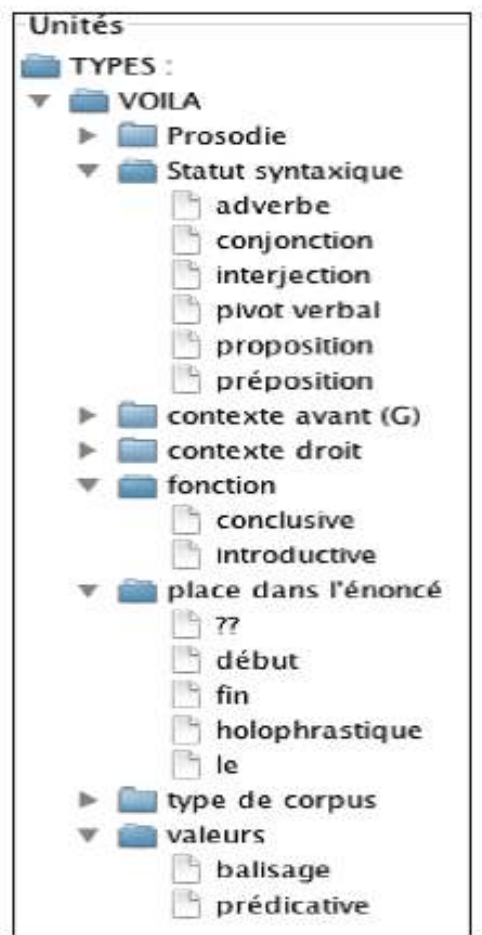

Fig. 1 : schéma d'annotation sous Analec

4 Les critères retenus pour l'analyse sont syntaxiques, «fonctionnels » et sémantiques et sont le reflet de ce que nous avons relevé dans nos lectures et nos premières observations.

5 Les statuts syntaxiques contiennent une sélection des différentes catégories rencontrées :

- Adverbe : ce statut correspond au cas où voilà porte sur une prédication entière et reprend un élément du contexte linguistique ; « Eh bien voilà, c'est pas mieux comme ça ? »

- Interjection : le cas de voilà interjection présente deux caractéristiques : une tendance à être holophrastique ou tout du moins en position détachée, et une fonction discursive et intersubjective marquée ; " tu vas t'asseoir ici et je vais te chercher quelque chose, voilà »

- Pivot verbal : voilà est analysable comme un pivot verbal quand il se rapproche le plus du verbe " voir " employé sans sujet. De manière plus générale, voilà comme pivot verbal marque une mise en relation d'une propriété avec une entité ; « --Eh bien!... me voilà propre... Il ne me manquait plus que cela... »

- Préposition : voilà introduit une information de type circonstant, souvent une mesure spatio-temporelle ; « Unifiée voilà deux décennies, la République fédérale d’Allemagne (RFA), quatrième puissance mondiale, est la première de l'Union européenne »

6 Les « fonctions " reflètent les différents rôles que joue voilà dans la construction et l'évolution de la scène verbale. Par " scène verbale ", nous entendons l'espace intersubjectif évoquée par la parole et qui se met en place au fur et à mesure que le discours se déroule (Victorri 1999). Une scène verbale, en tant que simulation qui se construit dans le déroulement du discours et qui reflète la compréhension du langage (Barsalou et al. 1993), a fondamentalement la propriété d'être perceptuelle et de " montrer » (ou " rendre présents » selon l'étymologie de "représenter ») des entités, des procès et leurs relations. Une scène est construite par et avec le discours et de ce 
point de vue, les unités linguistiques jouent toutes un rôle structurant (Col, en prep.). Pour ce qui est de voilà, nous avons retenu deux grandes fonctions :

- fonction introductive (voilà sert à introduire des entités et des procès sur la scène verbale) :

«Voilà une publicité dont l'éditeur d'antivirus (...) se serait sans doute bien passée »

- fonction conclusive (voilà sert globalement à clore une scène) : « Voilà pour la théorie. Dans

la pratique,... »

Pour ce qui est des critères sémantiques, nous avons opté pour une répartition binaire également et décidé de distinguer une valeur de balisage (introduction d'un repère dans le discours, donc un rôle structurant pour la scène verbale) et une valeur prédicative lorsque voilà sert à associer des propriétés à des entités, donc un rôle structurant pour le contenu informationnel. Cette bipartition est, comme les autres critères, provisoire et sera éventuellement revue lors d'analyses de données supplémentaires :

- balisage (structuration du discours) : « là, voilà, tu seras bien ». Cette valeur de balisage se manifeste quand voilà sert à délimiter des " régions» sur la scène verbale (souvent des moments-clé de la construction de la scène) qu'il rend plus ou moins saillantes.

- prédicative (structuration du contenu informationnel) : «voilà pourquoi le juge Lambert demande le renvoi ». Cette valeur prédicative se manifeste quand voilà sert à associer par exemple une cause avec un procès ou à introduire un élément sur la scène : « Enfin, me voilà en Normandie, au Mesnil-Roy. ».

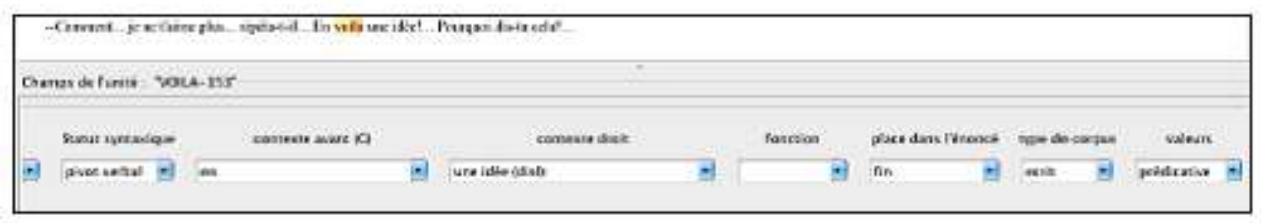

Fig. 2 : annotation d'un exemple

\section{Observations}

\subsection{Observations générales}

La première observation générale fournie par le logiciel Analec est celle de la répartition entre les deux types de corpus, écrit textuel et oral transcrit. Chacun de ces deux types partagent des propriétés spécifiques ce qui crée une différence nette entre eux. Il y a cependant davantage de variété dans le corpus " oral " (plus grande répartition géométrique) et donc une plus grande gamme de statuts syntaxiques, de valeurs et de fonctions, comme le montre la figure $3^{8}$ : 


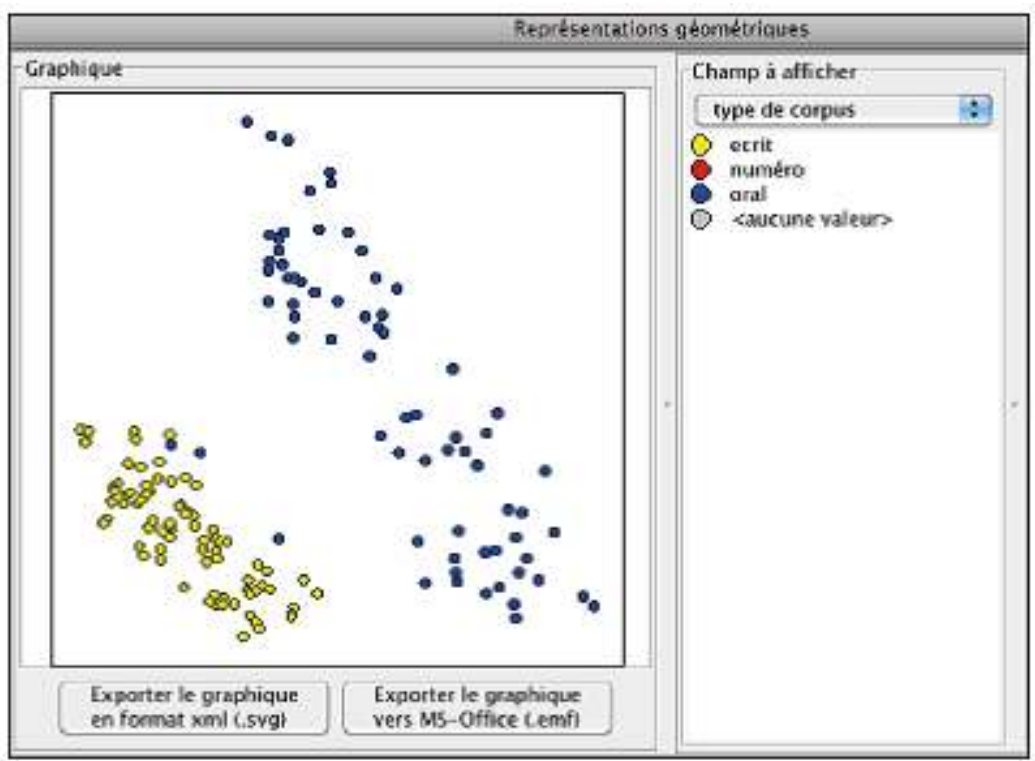

Fig. 3 : répartition entre types de corpus

Dans chaque groupe d'occurrences («oral » vs. écrit) se dégage néanmoins une valeur dominante : balisage pour la partie orale et valeur prédicative pour la partie écrite :

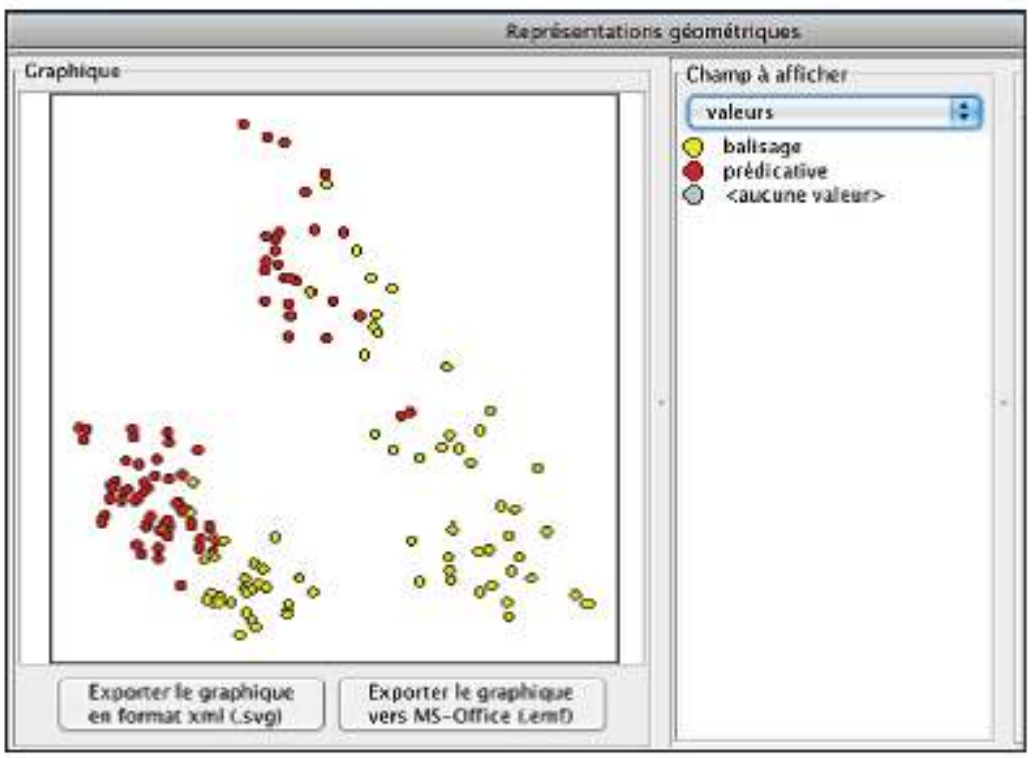

Fig. 4 : répartition entre valeurs

Quant aux fonctions de voilà, le rôle introducteur est plus dominant que le rôle conclusif, et ce dans les deux corpus, mais cette différence est davantage marquée dans 


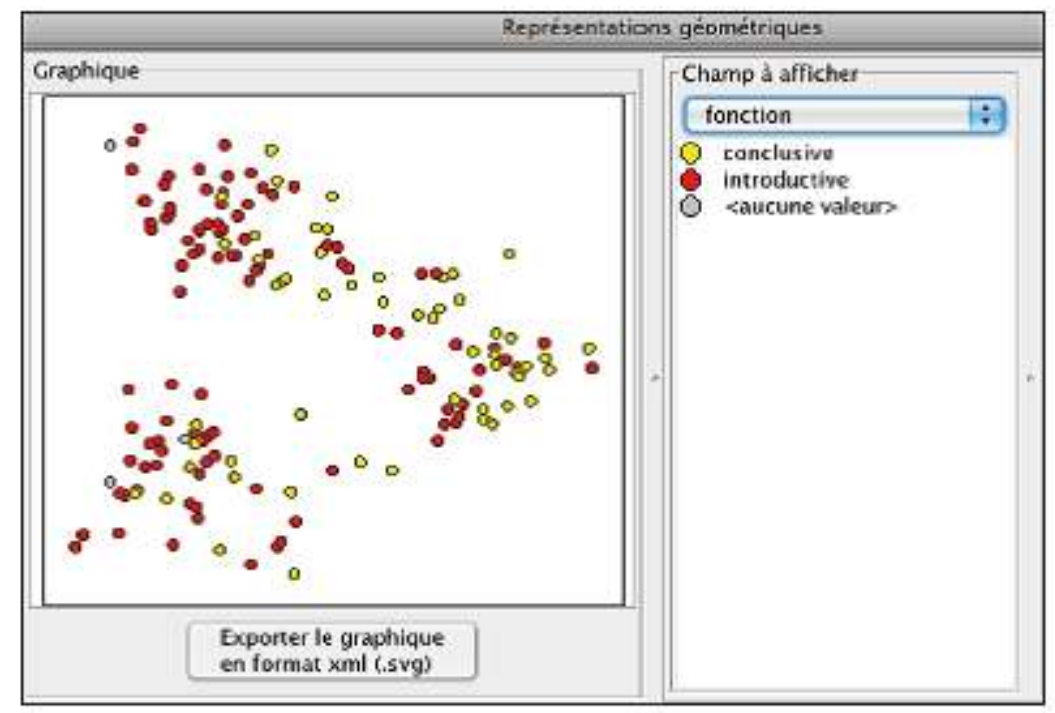

Fig. 5 : répartition entre fonctions

La grande répartition des structures syntaxiques rencontrées dans nos observations nous amène à les présenter sous forme de tableau (voir Annexe 2 : Structures cotextuelles) afin de développer ce qui nous parait plus fondamental pour notre objectif de cartographie : les valeurs typiques et les corrélations. Les propriétés typiques de chacun des types de corpus suivent la différence de corpus. Ce que nous appelons « propriétés typiques "sont les caractéristiques les plus pertinentes automatiquement produites par Analec. Ainsi dans le corpus « oral », et bien que la répartition soit plus harmonieuse que dans les autres parties du corpus, on note que la valeur de balisage se distingue, même très faiblement, par rapport à la fonction introductive et à la propriété de la place dans l'énoncé qui se trouve être à une faible majorité le début. La partie écrite de notre corpus contenant davantage d'occurrences, elle propose assez logiquement une plus grande variété de propriétés typiques. Nous voyons alors apparaître par exemple le critère de place dans le syntagme, celui du statut syntaxique et enfin celui de la complémentation. C'est donc un ensemble de propriétés plus important qui caractérise la partie " écrit » (c'est-à-dire hors oral transcrit). On relève bien entendu des différences entre les deux sous-types de corpus (le roman de Mirbeau et la presse) et le sous-type " presse » se distingue radicalement du fait de la présence 


\begin{tabular}{|l|c|c|c|c|c|c|}
\hline & $\begin{array}{c}\text { Complémen- } \\
\text { tation }\end{array}$ & Fonction & $\begin{array}{c}\text { Place dans } \\
\text { le syntagme }\end{array}$ & $\begin{array}{c}\text { Place dans } \\
\text { l'énoncé }\end{array}$ & $\begin{array}{c}\text { Statut } \\
\text { syntaxique }\end{array}$ & Valeur \\
\hline Oral & $\begin{array}{c}\text { sans } \\
(52.81 \%)\end{array}$ & $\begin{array}{c}\text { introductive } \\
(55.06 \%)\end{array}$ & - & $\begin{array}{c}\text { début } \\
(53.93 \%)\end{array}$ & - & $\begin{array}{c}\text { balisage } \\
(60.67 \%)\end{array}$ \\
\hline $\begin{array}{l}\text { Ecrit : } \\
\text { général }\end{array}$ & $\begin{array}{c}\text { avec } \\
(80.99 \%)\end{array}$ & $\begin{array}{c}\text { introductive } \\
(75.35 \%)\end{array}$ & $\begin{array}{c}\text { début } \\
(56.34 \%)\end{array}$ & $\begin{array}{c}\text { début } \\
(54.23 \%)\end{array}$ & $\begin{array}{c}\text { pivot verbal } \\
(56.34 \%)\end{array}$ & $\begin{array}{c}\text { prédicative } \\
(74.65 \%)\end{array}$ \\
\hline $\begin{array}{l}\text { Ecrit : } \\
\text { Mirbeau }\end{array}$ & - & $\begin{array}{c}\text { introductive } \\
(70.15 \%)\end{array}$ & - & $\begin{array}{c}\text { début } \\
(56.72 \%)\end{array}$ & $\begin{array}{c}\text { pivot verbal } \\
(53.73 \%)\end{array}$ & $\begin{array}{c}\text { prédicative } \\
(70.15 \%)\end{array}$ \\
\hline $\begin{array}{l}\text { Ecrit : } \\
\text { presse }\end{array}$ & - & $\begin{array}{c}\text { introductive } \\
(76 \%)\end{array}$ & - & début $(76 \%)$ & pivot verbal \\
$(58.67 \%)$ & $\begin{array}{c}\text { prédicative } \\
(78.67 \%)\end{array}$ \\
\hline
\end{tabular}

Tableau 1 : propriétés typiques du corpus et de ses différentes parties.

Le tableau ci-dessus correspond à l'analyse détaillée des sous-types du corpus (oral, écrit général, écrit Mirbeau et écrit presse) en fonction des critères retenus dans l'annotation du corpus. Les données chiffrées représentent les propriétés typiques produites par Analec, c'est à dire les fonctions, valeurs, etc les plus pertinentes dans l'analyse. Seules les propriétés les plus pertinentes apparaissent dans le tableau. Si une case est vide, c'est qu'aucune propriété n'a été retenue. Par exemple, dans la colonne " Statut syntaxique», aucune propriété n'émerge pour la partie orale du corpus ; c'est donc que la question du statut n'est pas suffisamment pertinente pour analyser ce type de d'énoncés (il y a trop de dispersion dans les résultats). On note aussi qu'un critère comme " Place dans le syntagme " s'avère guère pertinent ; il ne concerne finalement que l'écrit en général et du coup il est peu discriminant. Lors de la révision du schéma d'annotation pour analyser de plus amples données, ce critère pourra être enlevé. Le critère "Place dans l'énoncé » en revanche a plus de pertinence dans l'analyse et permet une caractérisation plus fine. Il ressort enfin de ce tableau que la valeur de Balisage et le critère "sans complémentation » sont deux propriétés typiques de l'oral et permettent une distinction robuste entre les deux corpus.

\subsection{Corrélations}

Les croisements de propriétés, ou " corrélations ", sont en fait très significatives et font émerger trois dimensions qui, bien qu'encore assez proches, donnent les premiers éléments de cartographie de voilà. La première dimension est celle de la place de voilà. Cette unité apparaît effectivement plutôt en début de syntagme ou d'énoncé et cette place est en congruence avec à la fois sa valeur dominante (valeur prédicative) et sa fonction dominante (introductive) comme le montre l'exemple suivant qui cumule ces trois propriétés :

(1) les Villemain sont à nouveau réunis mais voilà que le juge Simon semble basculer à son tour dans la malédiction de la Vologne : place initiale; valeur prédicative; fonction introductive

12 Les éléments introduits peuvent être situés à gauche de voilà (« me voilà en Normandie ») mais la plupart du temps, ils sont positionnés à sa droite (voir section 3). La 
corrélation fonctions/valeurs fait aussi apparaitre que voilà a la particularité d'avoir par ailleurs une valeur qui, même si elle est minoritaire et ne faisant pas partie des propriétés typiques, est corrélée de manière presque égale avec les deux fonctions conclusive et introductive ; c'est la valeur de balisage. Dit autrement, son rôle de balisage est relativement indépendant de ses fonctions (pas de corrélation forte) mais il est lié, on va le voir, avec un statut syntaxique particulier, celui d'interjection. La spécificité de l'interjection, pour ce qui est de voilà en tout cas, est d'être holophrastique :

(2) « là, voilà, là tu vas être très bien et les journalistes ne viendront pas t'y chercher "

13 À ce titre, la place de début se confond avec la place finale dans l'énoncé ou le syntagme, ce qui renforce finalement la tendance générale d'être en position initiale. La seconde dimension importante est celle de la valeur, en l'occurrence la valeur prédicative. Un lien intéressant est en fait apparu dans nos analyses entre le statut de pivot verbal et celui de la fonction introductive.

(3) Ah! les bureaux de placement, en voilà un sale truc... D'abord, il faut donner dix sous pour se faire inscrire; ensuite au petit bonheur des mauvaises places...

Si voilà sert à introduire des éléments sur la scène verbale, il sert aussi à associer des propriétés à ces éléments à travers une structure régulière : [voilà + prédication] comme dans :

(4) Non, non... pour rien au monde, je ne tolérerais un enfant chez moi... Ainsi, vous voilà prévenus... Arrangez-vous... prenez vos précautions...

15 La corrélation fonction/statuts fait aussi apparaître un rôle de voilà peu décrit, celui d'adverbe, dans lequel les fonctions conclusive et introductive sont équitablement partagées :

(5) Le populisme, voilà l'ennemi !

(6) ton père a une maîtresse voilà.

16 La dernière dimension apparaît dans la corrélation entre la valeur dominante de voilà (valeur prédicative) liée au statut de pivot verbal et la valeur de balisage liée au statut d'interjection. Ces corrélations donnent à voilà une fonction importante que l'on a déjà mentionnée plus haut : la fonction introductive. Mais cette fonction, quand on étudie de près les corrélations valeur/statuts syntaxiques, nous conduit à distinguer deux groupes de caractéristiques pertinentes :

- valeur de balisage ; statut d'interjection

- valeur prédicative ; statut de pivot verbal

17 L'observation des corrélations valeur / fonctions / statut syntaxique sur le corpus entier fait apparaitre outre trois dimensions importantes du « relief sémantique » de voilà, une amorce de dissociation entre deux groupes de caractéristiques. C'est cependant à travers une approche multi-échelle que l'on peut analyser un système complexe comme une langue naturelle (Col et Poibeau 2013). C'est pourquoi nous avons étendu ces premières analyses à un plus grand ensemble de données, et par ailleurs, nous avons recherché des régularités à un autre niveau, ce qui nous a permis de mettre en valeur d'autres dimensions de voilà. 


\subsection{Extension des données}

18 À l'aide de cette grille établie à partir d'un premier échantillonnage de voilà, nous avons recueilli 1065 exemples supplémentaires, tous issus de la base de données lexicale lexique.org. L'objectif de cette seconde étape est de mettre en évidence des patterns réguliers à une autre échelle que dans les analyses précédentes. Le relevé des unités qui figurent dans le contexte droit de voilà présentant une trop grande dispersion (ce qui par ailleurs renforce sa fonction introductive), nous nous sommes intéressés au contexte gauche. Nous avons surtout cherché à mettre en évidence des patterns très robustes contenant voilà. Ces patterns correspondent à des constructions au sens que leur donnent Goldberg $(1995,2006)$ et Croft $(2001)$ c'est-à-dire un assemblage d'unités récurrent et mêlant usage et abstraction. Les constructions ont effectivement un aspect symbolique (défendu par ailleurs en linguistique cognitive de Langacker à Fauconnier), mais elles sont aussi ancrées dans un usage et une régularité qui leur donnent une certaine robustesse c'est-à-dire une faible déformation (cf. Bybee 2007 ou Gries 2006). En dehors du critère de fréquence, ce qui définit une construction est aussi sa non réductibilité aux éléments qui la composent. On ne peut effectivement pas entièrement prédire le sens d'une construction à partir de ses seuls composants :

" Any linguistic pattern is recognized as a construction as long as some aspect of its form or function is not strictly predictable from its components parts or from other constructions recognized to exist. In addition, patterns are stored as construction even if they are fully predictable as long as they occur with sufficient frequency » (Goldberg 2006: 5)

Goldberg fait ainsi une distinction entre les constructions issues d'une grande fréquence comme par exemple le schéma VOL (Verbe - Objet - adjoint Locatif) pour le verbe anglais put (Goldberg 2006, 107-11) et les schémas non complètement décomposables comme celui des patterns causatifs de type [SUBJ [V OBJ OBL]] (cf le Caused Motion Construction de Goldberg 1995, chapitre 7). C'est le premier type de construction qui a retenu notre attention lors de l'analyse de données complémentaires. Une première construction robuste et très générale confirme à la fois la position de voilà en début de séquence (syntagme ou énoncé) et va dans le sens de sa fonction introductive. Nous avons effectivement relevé une très forte proportion de [Voilà (...)] dans lexique.org (96,33\%). Ce taux important tient sans doute au recueil des données présentes dans la base (choix de ne prendre que des voilà en début d'énoncé ?) et à leur classement, mais il confirme malgré tout, les observations effectuées sur nos propres données. Il n'en reste pas moins que ce premier pattern, tout robuste qu'il soit, doit être affiné. C'est en fait deux constructions plus « nuancées » qui ont retenu notre attention, qui vont chacune dans le sens de la dissociation déjà observée entre balisage + interjection / valeur prédicative + pivot verbal : [voilà + pause] (44\%) et [voilà + entité/ procès] $(44,78 \%)$.

La première construction se subdivise en fait en deux structures régulières :

211 . <...] voilà.>, c'est-à-dire le cas où l'unité est suivie d'une pause « définitive », c'est-àdire par un silence de 0.2 seconde minimum intervenant au terme d'un énoncé ou d'un tour de parole, la fonction conclusive étant généralement corrélée par l'intonation finale $(26,85 \%)$ :

(7) en attendant tu vas t'asseoir ici et je vais te chercher quelque chose voilà. 
2. <voilà, [...]> c'est-à-dire le cas où l'unité est suivie d'une pause relative, c'est-à-dire un silence de longueur variable qui peut être due à des phénomènes physiologiques (respiration), sémantique (effet rhétorique) ou cognitive (recherche lexicale notamment (Zellner 2010, Di Cristo 2013) (17,18\%) :

(8) Mais voilà, il est depuis recherché par la police dans le cadre d'une enquête sur une affaire de meurtre.

La seconde construction correspond de son côté à trois cas différents qui ont en commun d'introduire sur la scène une entité (ou des entités) ou un procès " partagés ». Ce que nous appelons des entités ou des procès partagés sont des éléments à propos desquels locuteurs et co-locuteurs ont des connaissances communes, que ces connaissances soient contextuelles (à l'échelle du discours) ou qu'elles relèvent davantage de la mémoire des sujets impliqués dans le discours. Nous relevons ainsi une récurrence notable de structures où l'entité est antéposée ou postposée, mais en tout cas introduite par un article défini ou par un pronom : d'une dispersion apparente de constructions et d'emplois, font émerger des constructions relativement robustes et avec elles des routines cognitives pertinentes. En effet, ces patterns font apparaître au moins deux rôles complémentaires de voilà : introduire des éléments partagés sur la scène verbale en cours de déroulement (voilà associé aux articles définis et aux pronoms), et d'autre part, délimiter une scène (voilà associé à une prédication). Ces deux rôles vont servir de pistes pour une hypothèse sur la définition instructionnelle de voilà et sur sa participation à la construction du sens d'un énoncé contenant cette unité.

(9) Zumba, zumba, me voilà !

(10) -- Nous voilà donc entrés dans ce qu'on pourrait appeler un régime d'austérité sub-atroce, en fait le pire de tous, en tout cas du point de vue du « hors système »les populations.

II. entité postposée :

(11) Déjà là ça ça donne lieu à quelques quiproquos ce qui permet que les autres euh réajustent le tir et euh précisent en disant bah voilà voilà les circonstances voilà les personnages voilà euh l'histoire en gros bon

III. procès postposé :

(12) Ce ne sont pas des chartes qui nous délivreront de ça, voilà pourquoi il importe de construire autre chose.

(13) Le CAC40 remonte, voilà comment en profiter!

(14) Une allégorie de l'enfer : voilà ce que pourrait être le delta du Niger depuisque l'exploitation du pétrole a pris le contrôle des terres des Ogonis.

(15) voilà qui devrait conférer un socle de réflexion moins discutable que le sont les nombreux sophismes entendus sur cette question délicate 


\section{Pistes pour une hypothèse}

31 La cartographie de voilà qui se dessine à travers des analyses menées sur un échantillon relativement important d'exemples (1286 au total) et avec une grille d'analyse contenant des critères autant syntaxiques, que sémantiques et fonctionnels donne finalement peu d'explications pour rendre compte du caractère expansif de cette unité discursive. Si nous ne disposons pour l'instant que de peu d'éléments d'explication de son succès, nous avons pu cependant observer deux structures sémantico-cognitives en apparence "concurrentes » et correspondant à deux rôles joués par voilà dans la structuration et l'évolution de la scène verbale construite par un énoncé : introduire des éléments sur la scène et baliser la scène. On peut supposer que si ces deux structures étaient réellement en concurrence, l'expansion de voilà serait entravée et donc freinée car ces deux rôles seraient opposés. On peut alors plutôt parler de deux rôles complémentaires, exercés à deux niveaux différents. Un de ces rôles s'effectuerait à un niveau plus fondamental que le second : introduire des éléments sur une scène verbale et en conséquence, esquisser les contours de la scène en cours de constitution. Ce rôle correspond aux trois grandes dimensions relevées à partir des valeurs typiques (cf. Tableau 1) et des corrélations des différentes propriétés de voilà (cf. 3.2.) : position initiale, valeur prédicative et bien entendu fonction introductive. Le second rôle se dégage d'autres propriétés et il est davantage spécifique du corpus oral : baliser la scène verbale et la faire évoluer vers une autre scène. Ce rôle correspond alors à son statut récurrent d'interjection (et donc son isolement dans l'énoncé) autant qu'à sa valeur de balisage, et enfin qu'à sa fonction conclusive certes non majoritaire mais malgré tout importante ( $45 \%$ des occurrences de la partie orale du corpus). Afin de rendre compte de la complémentarité des deux rôles de voilà, le recours à une hypothèse reposant sur des éléments fonctionnels peut sembler pertinent et utile. Si on part du principe que voilà se répand facilement et assez vite dans l'usage langagier quotidien, il doit y avoir des facteurs facilitants liés à nos activités cognitives générales. Voilà répondrait ainsi à un besoin de regrouper des informations et des éléments de la scène verbale en un ensemble cohérent et évolutif. Sa double activité introductive et conclusive permettrait tour à tour de proposer une gestalt sémantique aux participants $\mathrm{du}$ discours. Nous faisons ici une analogie avec notre capacité décrite en psychologie gestaltiste (chez Köhler, Wertheimer ou Guillaume) de percevoir des éléments de manière regroupée, comme une configuration cohérente (à proprement parler, une " gestalt »). Les travaux de Moore et Egeth (Moore et Egeth 1997) vont jusqu'à montrer que dans le domaine de la perception visuelle, le regroupement se fait sans attention particulière même si des travaux plus récents comme ceux de Palmer (Palmer 2002) nuancent cette position et montrent que le regroupement s'effectue à un niveau de conscience plus élevé. Le regroupement servirait en fait à créer une sorte d'attention, au moins visuelle. Au niveau langagier, nous pouvons suggérer que voilà serait une unité dont le rôle essentiel serait de regrouper des entités et des procès en vue de maintenir une forme d'attention et par là même une forme de compréhension à un niveau satisfaisant et relatif (compréhension dite " good enough » après un traitement partiel et incomplet de l'information, Ferreira, Bailey et Feraro 2002). C'est en définitive sur un plan procédural et instructionnel que nous proposons de formuler des éléments d'hypothèse. Nous privilégions effectivement une approche dynamique de la construction du sens qui considère qu'une unité linguistique contribue au sens de l'énoncé lors de son traitement en même temps qu'elle est elle-même déterminée par 
les autres unités présentes dans le contexte et qu'elle a convoquées. C'est dans ce double mouvement de construction sémantique qu'une unité linguistique doit être comprise et c'est à partir de ce principe de convocation-évocation développé par Victorri (1999) et Col (Col 2011, Col, Aptekman, Girault, Poibeau 2012, Col et Poibeau 2014) que nous souhaitons formuler des éléments de l'instruction sémantique fournie par voilà. Cette instruction aurait ainsi deux aspects complémentaires et unifiés : intégrer des entités ou des procès dans une représentation partagée sur la scène verbale et déclencher le regroupement de ces entités et de ces procès lors de leur intégration dans la représentation partagée. Voilà contribuerait ainsi à faire un " paquet " d'informations pour ainsi dire et à faire évoluer ce paquet vers une autre scène verbale. Voilà marquerait le passage d'une information subdivisée (Fauconnier 1997, Dinsmore 1991) à une information regroupée. Cette hypothèse aurait ainsi le mérite de tenir compte des trois grandes dimensions apparues dans nos analyses sur corpus, ainsi que des différents patterns également relevés dans nos observations. Elle présente aussi l'avantage d'être cohérente avec la définition que propose Hansen 1997 des «marqueurs discursifs": ils ne contribuent pas directement au contenu propositionnel mais ils fonctionnent comme des instructions qui explicitent comment intégrer ces unités dans une représentation mentale cohérente du discours (Hansen, 1997 : 161). En définitive, voilà serait une unité qui convoque sur la scène verbale des éléments dispersés (entités ou procès) et elle évoquerait leur regroupement dans un ensemble perceptible.

\section{Conclusion}

Pour répondre à la question : « qu'est-ce qui facilite l'expansion de voilà ? ", nous avons choisi de nous consacrer tout d'abord à la description de son comportement dans un petit corpus. Le choix de la taille répond à une exigence méthodologique : nous souhaitions avant tout mettre en évidence une grille de lecture avant d'aborder des données plus vastes. Ces données ont par ailleurs été en partie abordées dans cette étude ${ }^{9}$. Nous sommes maintenant en mesure de faire ces nouvelles analyses, et nous les avons même déjà entamées dans cette étude. Pour la composition du corpus, nous avons choisi d'associer de l'écrit et de l'oral dans le but d'affiner dès le départ les critères d'analyse en respectant une vraie continuité entre français écrit et français parlé. Cette approche nous a permis de faire apparaître deux grandes propriétés complémentaires de voilà qui seraient chacune apparues séparément et sans complémentarité : sa valeur de balisage associée à son statut d'interjection d'une part, et sa valeur prédicative associée à son statut de pivot verbal d'autre part. Cette double propriété se voit confirmée dans l'analyse de données plus vastes à travers la recherche de constructions et de patterns robustes. Nous avons mis en évidence en effet deux patterns réguliers représentés en proportions quasiment équivalentes : [voilà + pause] et [voilà + entité/procès]. A partir de cette mise en évidence, nous suggérons une hypothèse cognitive qui vise à donner à voilà une fonction importante dans la création et le maintien de l'attention dans le discours. Cette hypothèse, liée à la capacité de regroupement développée dans le domaine de la perception, s'appuie sur un travail en cours et se trouve seulement ébauchée ici. Elle devrait être développée conjointement à l'exploration de sous-structures linguistiques à partir des deux principaux patterns que nous avons déjà relevés. 


\section{BIBLIOGRAPHIE}

Anscombre, J.-C. (1990). « Pourquoi un moulin à vent n'est pas un ventilateur », Langue française $86,103-125$

Anscombre, J.-C. (2001). «Le rôle du lexique dans la théorie des stéréotypes », Langages $\mathrm{n}^{\circ} 142$ : 57-75.

Auchlin, A. (1981). « Mais heu, pis bon, ben alors voilà, quoi ! Marqueurs de structuration de la conversation et complétude. Cahiers de linguistique française 2, 141-160.

Barsalou, L., Yeh, W., Luka, B., Olseth, K., Mix, K. \& Wu, L.-L. (1993). « Concepts and meaning ». In Chicago Linguistics Society 9: Papers from the Parasessions on Conceptual Representations, Volume 2, K. Beals, G. Cooke, D. Kathman, K. E. McCullough, S. Kita \& D. Testen (eds), 23-61. Chicago Linguistics Society.

Bergen, B. et Plauché, M. (2001). « Voilà, voilà: Extensions of Deictic Constructions in French », in A. Cienki, B. Luka et M. Smith (eds) Conceptual and Discourse Factors in Linguistic Structure, CSLI. Brunot, F., Bruneau, C. (1969). Précis de grammaire historique de la langue française. Masson et Cie, Paris.

Bybee, J. (2007). Frequency of use and the organization of language. Oxford: Oxford University Press. Col, G. (2011). « Modèle instructionnel du rôle des unités linguistiques dans la construction dynamique du sens ", in Le Langage et ses niveaux d'analyses. J. Chuquet (dir). Rennes, Presses Universitaires, 45-60.

Col, G. (en prep). Sémantique instructionnelle. Contribution à l'étude de la construction dynamique du sens. (ms)

Col, G., Aptekman, J. Girault, S. et Poibeau, T. (2012). « Gestalt Compositionality and Instructionbased Meaning Construction ». Cognitive Processing, Vol. 13, issue 2, pages 151-170.

Col, G. et Poibeau, T. (2013). « Du continu dans les interactions entre unités linguistiques », Conférence internationale «Interactions dans les Systèmes Complexes », Orléans

Col, G. et Poibeau, T. (2014). « An Instruction-Based Analysis of Over », Language and Cognition, vol. 6., num. 3, pp 370 - 407.

Croft, W. (2001). Radical Construction Grammar: syntactic theory in typological perspective. Oxford: Oxford University Press.

Damourette, J., Pichon, E. (1911-1940). Des mots à la pensée, vol. 7. Edition d'Artrey, Paris.

Dauzat, A. (1947). Grammaire raisonnée de la langue française. Myon, Editions I.A.C.

De Cesare, A.-M. (2011). « L'italien ecco et les français voici, voilà. Regards croisés sur leurs emplois dans les textes écrits ", Langages, 184, p. 51-67

Delahaie, J. (2009). « Voilà le facteur ou voici le facteur ? Etude syntaxique et sémantique de voilà ", Cahiers de lexicologie. 95-2, p. 43-58

Dervillez-Bastuji, J., (1982). Structures des relations spatiales dans quelques langues naturelles. Introduction à une théorie sémantique. Genève : Droz

Di Cristo, A. (2013). La Prosodie de la parole. Bruxelles : De Boeck

Dinsmore, J. (1991). Partitioned Representations. Dordrecht : Kluwer 
Druetta, R. (1993). Etude de voilà non-constructeur, tesi di Laurea, Universita di Torino, manuscrit dactylographié.

Fauconnier, G. (1997). Mappings in Thought and Language. Cambridge : Cambridge University Press Ferreira, F., Bailey, K., Ferraro, V. (2002). « Good-Enough Representations in Language Comprehension. », Current Directions in Psychological Science, Vol. 1, Issue 1, pages 11-15 Girault-Duvivier, C. P. (1840). Grammaire des grammaires. Paris, Cotelle.

Goldberg, A. (1995). Constructions: A Construction Grammar Approach to Argument Structure. University of Chicago Press.

Goldberg, A. (2006). Constructions at Work : the nature of generalization in language. Oxford University Press.

Grenoble, L. et Riley, M. (1996). « The role of deictics in discourse coherence : French voici/voilà and Russian vot/von ", Journal of Pragmatics 25

Grevisse, M. (1980). Le Bon usage. Duculot.

Gries, S. (2006). " Corpus-based methods and cognitive semantics: the many meanings of to run ». In Gries S. \& Stefanowitsch A. (eds.), Corpora in Cognitive Linguistics: Corpus-Based Approaches to Syntax and Lexis. Berlin \& New York : Mouton de Gruyter. 57-99.

Guillaume, P. (1979). La Psychologie de la forme. Paris, Flammarion (réed).

Hansen, M.-B. M. (1997). « Alors and donc in spoken French: A reanalysis », Journal of Pragmatics 28,153-187

Köhler, W. (1929). Gestalt Psychology. New York, Liveright

Landragin, F., Poibeau, T., Victorri, B. (2012). « ANALEC: a New Tool for the Dynamic Annotation of Textual Data ", Proceedings of the International Conference on Language Resources and Evaluation (LREC 2012), Istanbul, Turkey

Léard, J.-M. (1996). « Ti / -tu, est-ce que, qu'est-ce que, ce que, hé que, don: des particules de modalisation en français? ", Revue québécoise de linguistique, vol. 24, n² 2, 1996, p. 107-124.

Le Bidois, G. (1965). Syntaxe du francais moderne Ses fondements historiques et psychologigues. Paris, Editions Auguste Picard.

Moignet, G. (1969). « Le verbe voici - voilà », Travaux de linguistique et de littérature, 8, 1, p. 189-202.

Moore, C., M., Egeth, H. (1997). « Perception Without Attention: Evidence of Grouping Under Conditions of Inattention », Journal of Experimental Psychology, Vol. 23, No. 2, 339-352

Morin, Y, C. (1985) « On the two french subjectless verbs 'voici' and 'voilà' », Language, vol.61-4, p. 777-820

Narjoux, C. (2003). « C'est cela que c'est la tragédie » ou les présentatifs dans Electre de Giraudoux ", Information grammaticale, 96

Nølke, H. (1994). Linguistique modulaire : de la forme au sens. Bibliothèque de l'Information Grammaticale, Peeters, Paris, Louvain

Nyrops, C. (1914). Grammaire historique de la langue française (1914-1930). Slatkine.

Oppermann-Marsaux, E. (2006). « Les origines du présentatif voici/voilà et son évolution jusqu'à la fin du XVIe siècle », Langue française 149, p. 77-91. 
Palmer, S. (2002). «Perceptual Grouping: It's Later Than You Think », Current Directions In Psychological Science, vol. 11, num. 3, 101-106

Petit, M. (2009). Discrimination prosodique et représentation du lexique : application aux emplois des connecteurs discursifs. Thèse de Doctorat, Université d'Orléans.

Porhiel, S. (2012). « The presentative voici/voilà - Towards a pragmatic definition ». Journal of Pragmatics 44, 435-452

Pourquery D. (2014). Les Mots de l'époque. 100 tics, trouvailles et autres extravagances du langage quotidien. Autrement.

Riegel, M., Pellat, J.-C., Rioul, R. (1994). Grammaire méthodique du français. PUF, Paris.

Victorri, B. (1999). « Le sens grammatical », Langage 136, 85-105

Wagner, R.-L. et Pinchon, J. (1962). Grammaire du français classique et moderne. Hachette.

Wertheimer, M. (1950). « Laws of organization in perceptual forms ». In W.D. Ellis (Ed.), A sourcebook of Gestalt psychology (pp. 71-81). New York: The Humanities Press.

Zellner, B. (1994). « Pauses and the temporal structure of speech ». In E. Keller (Ed.), Fundamentals of Speech synthesis and speech recognition. Chichester : John Wiley, 41-62

\section{ANNEXES}

Annexe 1 : Tableau synoptique des approches de voilà

\begin{tabular}{|c|c|c|c|c|}
\hline Anteurs & Statut Catégoriel & Sémantique & \begin{tabular}{|l} 
Pragmatique I \\
Discours
\end{tabular} & \begin{tabular}{|l|} 
Enonciation / \\
Textualisation
\end{tabular} \\
\hline $\begin{array}{l}\text { Wagner (1966) } \\
\text { /Wagner et } \\
\text { Pinchon (1991) }\end{array}$ & $\begin{array}{l}\text { Particule } \\
\text { démonstrative }\end{array}$ & & & \begin{tabular}{|l|} 
Sorte d'iruption \\
(im) prévevie dans \\
lactualité du \\
locuteur ou de son \\
allocutaire
\end{tabular} \\
\hline \begin{tabular}{|l|} 
Brunot et \\
Bruneau (1969)
\end{tabular} & $\begin{array}{l}\text { Adverbe } \\
\text { présentarif }\end{array}$ & & & \\
\hline \begin{tabular}{|l} 
Moignet \\
$(1969 / 1974)$
\end{tabular} & 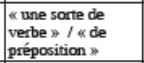 & & & \\
\hline $\begin{array}{l}\text { Auchlin } \\
\text { (1981) }\end{array}$ & $\begin{array}{l}\text { Marqueur de } \\
\text { structuration de } \\
\text { conversation }\end{array}$ & & $\begin{array}{l}\text { MSC à valeur } \\
\text { de clôture- } \\
\text { conclusion }\end{array}$ & $\begin{array}{l}\text { Délimitation, } \\
\text { liaisond des énoncés } \\
\text { Lié au niveau de } \\
\text { textralisation }\end{array}$ \\
\hline \begin{tabular}{|l|} 
Dervillez- \\
Bastuji (1982) \\
\end{tabular} & \begin{tabular}{|l|}
$\begin{array}{l}\text { Présentateurs } \\
\text { déictiques }\end{array}$ \\
\end{tabular} & & & $\begin{array}{l}\text { Relève de la déixis } \\
\text { spatiale }\end{array}$ \\
\hline $\begin{array}{l}\text { Morin } \\
(1985)\end{array}$ & subjectless verb & & & \\
\hline $\begin{array}{l}\text { Gardes-Tamine } \\
\text { (1986) }\end{array}$ & $\begin{array}{l}\text { Le plus } \\
\text { démonstratif des } \\
\text { présentatifis }\end{array}$ & & & $\begin{array}{l}\text { Liué à l'énonciateur. } \\
\text { rivé à situation } \\
\text { d'énonciation }\end{array}$ \\
\hline $\begin{array}{l}\text { Leard } \\
\text { (1992) }\end{array}$ & \begin{tabular}{|l|} 
verbe / voilà + + \\
notion verbale / \\
prépositionnel / \\
marqueur discursif
\end{tabular} & \begin{tabular}{|l|} 
« pointage à \\
partir du lieu ou \\
du moment de \\
la parole *
\end{tabular} & $\begin{array}{l}\text { voilà discursif : } \\
\text { valeur } \\
\text { géographique/ } \\
\text { illocutoire }\end{array}$ & $\begin{array}{l}\text { Valeur de base : } \\
\text { déictique et } \\
\text { aspectruelle }\end{array}$ \\
\hline \begin{tabular}{|l} 
Grenoble et \\
Riley (1996)
\end{tabular} & $\begin{array}{l}\text { Présentarifs; } \\
\text { deixis seconde }\end{array}$ & $\begin{array}{l}\text { Proposition: } \\
\text { portée } \\
\text { sémantique } \\
\text { ninimume }\end{array}$ & \begin{tabular}{|l|} 
Anaphorique \\
ou cataphorique \\
' gestion de \\
I ' interaction
\end{tabular} & \begin{tabular}{|l} 
Merqueur discursif \\
(texpe comume \\
espace)
\end{tabular} \\
\hline $\begin{array}{l}\text { Narjoux } \\
(2003)\end{array}$ & \begin{tabular}{|l|} 
Présentataif \\
prédicatif \\
oo à aleur \\
interjective, \\
prépositionnelle
\end{tabular} & & & 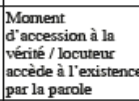 \\
\hline \begin{tabular}{|l|} 
Opperman- \\
Marsaux (2006)
\end{tabular} & Présentatif & & & \begin{tabular}{|l} 
Sitruationnel/ \\
Narratif / Texruel
\end{tabular} \\
\hline Delahaie (2009) & $\begin{array}{l}\text { Présentatif ou } \\
\text { conversationnel }\end{array}$ & & $\begin{array}{l}\text { Ouvre, ferme, } \\
\text { confinme. } \\
\text { Introduit une } \\
\text { stéréotypie } \\
\end{array}$ & $\begin{array}{l}\text { Ni présentatifif. } \\
\text { ni déictique : } \\
\text { Rapport érroit à } \\
\text { I'énonciation }\end{array}$ \\
\hline $\begin{array}{l}\text { De Cesare } \\
\text { (2011) }\end{array}$ & & $\begin{array}{l}\begin{array}{l}\text { Acte assertif : } \\
\text { pointagere. } \\
\text { présentation }\end{array} \\
\end{array}$ & & $\begin{array}{l}\text { «S Signal discursif } \\
\text { pour l'emploi seul }\end{array}$ \\
\hline $\begin{array}{l}\text { Porhiel } \\
\text { (2012) }\end{array}$ & \begin{tabular}{|l|} 
Préposition / \\
Présentatifif / \\
Marqueur de \\
discours
\end{tabular} & & & \\
\hline
\end{tabular}

Annexe 2 : Structures co-textuelles

GN + voilà 
(10) Nous voilà donc entrés dans ce qu'on pourrait appeler un régime d'austérité sub-atroce,

GN + voilà + pred :

(16) Eh bien!... me voilà propre... Il ne me manquait plus que cela...

$\mathrm{ADV}+$ voilà

(17) Seulement voilà, la guerre en Afghanistan a maintenant plus de onze ans d'âge conj. + adv. + voilà

(18) et bien voilà c'est pas mieux comme ça conj. + objet + voilà + pred.

(19) Et le voilà qui cause avec lui.

conj. + voilà

(20) Mais voilà, il est depuis recherché par la police dans le cadre d'une enquête sur une affaire de meurtre

conj. + voilà + GN :

(21) et voilà le travail $\mathrm{P}$ ici notre tablier est terminé conj. + voilà + préd.

(22) et voilà ils nous piquent notre BM tu vois je te l'avais dit

(1) les Villemain sont à nouveau réunis mais voilà que le juge Simon semble basculer à son tour dans la malédiction de la Vologne

préd. + voilà + GN

(23) Il écrivait, voilà près de soixante-dix ans, qu'« un moratoire sur la recherche atomique, tout en nous privant d'électricité bon marché, pourrait nous permettre de continuer à vivre sur une planète habitée ».

(24) Unifiée voilà deux décennies, la République fédérale d'Allemagne (RFA), quatrième puissance mondiale, est la première de l'Union européenne, la plus peuplée -82 millions d'habitants (2) - et la plus productive, avec un produit intérieur brut de 2407 milliards d'euros en 2009.

voilà $+\mathrm{GN}$

(25) CINÉMA • Oubliez Bollywood, voilà Mollywood !

(26) --Il ne n'aime plus, voilà mon malheur... Il ne m'aime plus...

voilà + GN + pred

(27) --Eh bien?... Que faites-vous donc?... Vous n'entendez donc pas?... Êtes-vous sourde?... Voilà trois heures que je sonne... C'est agaçant, à la fin...

voilà + pred.

(15) Le refus du déni sur la parenté biologique, l'obligation d'offrir à tout enfant une filiation symbolique ne reposant pas sur le déni ou le secret, et la latitude donnée à tout citoyen d'élever un enfant quelle que soit sa sexualité : voilà qui devrait conférer un socle de réflexion moins discutable que le sont les nombreux sophismes entendus sur cette question délicate.

(28) --Voilà ce que vous devriez faire, mademoiselle Célestine...

$\varnothing$ voilà $\varnothing$

(29) --Eh bien, Célestine... je vous trouve charmante... voilà!

(30) A--Ben sûr!... Faut se faire une raison...

B--Voilà!... 


\section{NOTES}

1. Pour leurs avis éclairés sur ce travail, les auteurs remercient les membres de la thématique Discours et Cognition (DisCo) du laboratoire FoReLL (Poitiers, MSHS).

Le document entier (session vidéo de Le Cam, en monologue face à sa caméra embarquée à bord) est accessible ici : http://www.dailymotion.com/video/xwx9dk_jean-lecam-raconte-sonparcours-du-combattant_sport.

2. Nous nous pencherons plus spécifiquement sur la mesure de son expansion dans une prochaine étude, à partir de corpus purement oraux comme le CFPP2000 qui propose un certain nombre de voilà qui semblent correspondre à l'usage qui se répand le plus aujourd'hui.

3. On sait notamment, grâce aux travaux d'Oppermann-Marsaux (2006), que la forme soudée de voilà apparaît au XIVe siècle (grammaticalisation sans doute) et que, dès le XVIe siècle, voilà peut être employé en mot phrase (emploi seul), ce qui place ses propriétés verbales au second plan. La grande polyvalence de voilà pourrait se comprendre à la lumière de cette désémantisation progressive.

4. Que l'on songe par exemple à la construction rarement envisagée mais très spécifique « voilà qui » où « qui » renvoie à un non-humain (" voilà qui est dangereux ", « voilà qui est bien », " voilà qui m'étonne ») : voilà semble être le seul contexte autorisant aujourd'hui l'emploi de " qui » avec un référent non-humain (à distinguer de "le voilà qui cause avec lui »-présence d'un antécédent - et de " voilà ce qui... ", " voilà de quoi occuper les gens » - relative substantive).

5. Un corpus plus vaste est en cours de constitution au sein du projet DisCo et sera orienté vers l'oral (voir note 2).

6. Des informations sur la thématique se trouvent ici : http://forell.labo.univ-poitiers.fr/ activites-scientifiques/thematiques-transversales/

7. http://www.lattice.cnrs.fr/Telecharger-Analec

8. Dans la figure 3, l'étiquette «numéro » renvoie aux numéros de chacun des exemples.

9. Les données orales seront abordées de manières plus approfondie dans une prochaineétude (voir note 2).

\section{RÉSUMÉS}

Éléments de cartographie des emplois de voilà en vue d'une analyse instructionnelle Voilà est une unité dont l'usage se répand rapidement en français oral aujourd'hui. Ce travail cherche à proposer une explication de cette expansion en mettant en évidence des facteurs de facilitation. A partir d'une description du comportement de l'unité en contexte (sur corpus oral et écrit), nous mettons en évidence des propriétés typiques qui sont ensuite testées sur des données supplémentaires. Des constructions et des patterns relativement robustes émergent également des analyses sur corpus. Voilà se caractérise par deux comportements essentiels ([VOILA + pause] et [VOILA + entités/procès]) et deux groupes de valeurs /statuts associés : valeur de balisage + statut d'interjection ; valeur prédicative + statut de pivot. Est alors proposée une hypothèse fonctionnelle qui repose sur l'activité cognitive de regroupement : voilà servirait à intégrer en les regroupant des informations sur une scène verbale. L'instruction sémantique donnée par voilà consisterait ainsi à convoquer des éléments sur une scène et à évoquer leur regroupement dans un ensemble perceptible. 
Tentative mapping of the various uses of voilà : toward an instructional analysis The lexical item voilà is fast spreading in contemporary spoken French. This article offers a possible explanation for this rapid expansion by identifying contributing factors. We first describe voilà's contextual behaviors in both written and spoken corpora. Typical properties arise from the description and are further tested on additional data. Constructions and relatively robust patterns emerge. Voilà is indeed characterized by two essential behaviors - [VOILA + pause] and [VOILA + entity/process] - and two groups of paired values and statuses - [signpost value + interjection status] and [predicative value + pivotal status]. We then formulate a functional hypothesis in terms of cognitive grouping: voilà would integrate informational elements by grouping them on the verbal scene. The semantic instruction given by voilà would consist in convoking elements on a scene and evoking their grouping into a perceptible set.

INDEX

Mots-clés : corpus écrit, corpus oral, polysémie, Constructions, instruction, scène verbale, regroupement

Keywords : written corpus, oral corpus, polysemy, Constructions, instruction, verbal scene, grouping

\section{AUTEURS}

\section{JULIEN RAULT}

Laboratoire FoReLL - EA 3816, Université de Poitiers 